SLAC-PUB-11052

March 2005

\title{
Resistive Wall Impedance of an Insert*
}

\author{
Gennady Stupakov \\ Stanford Linear Accelerator Center, Stanford University, Stanford, CA \\ 94309
}

\begin{abstract}
The standard theoretical formulae for resistive wall impedance are usually derived in a model which assumes an infinitely long pipe. In practice, one often has to deal with resistive inserts with a conductivity different from the rest of the pipe. To address this case, we calculate the resistive wall impedance when the wall conductivity varies along the axis of the pipe. We show that at not very high frequencies the impedance of an insert per unit length is given by the same formulae as for an infinitely long pipe.
\end{abstract}

Submitted to Physical Review Special Topics - Accelerators and Beams

*Work supported by Department of Energy contract DE-AC02-76SF00515. 


\section{Introduction}

The standard theoretical formulae for resistive wall impedance (see, e.g. [1]) are derived in a model of an infinitely long pipe. The longitudinal impedance per unit length of the pipe, in CGS system of units, is given by the following equation

$$
Z_{\text {long pipe }}(\omega)=\frac{1-i}{c b} \sqrt{\frac{\omega}{2 \pi \sigma}},
$$

where $\omega$ is the frequency, $b$ is the pipe radius, and $\sigma$ is the conductivity of the pipe wall. Eq. (1) is valid for not very large frequencies,

$$
\omega \ll \omega_{0},
$$

where $\omega_{0}=\left(4 \pi c^{2} \sigma / b^{2}\right)^{1 / 3}$. This condition is usually satisfied in practice, except for extremely short bunches (see discussion in [2]).

In practice, one often has to deal with resistive inserts with a conductivity different from the rest of the pipe. The question then arises whether Eq. (1) can be used in such cases. In this paper we calculate the resistive wall impedance when the wall conductivity varies along the axis of the pipe in the regime when condition (2) is satisfied. In Section 2 we study the case of a perfectly conducting round pipe with an insert of finite length $L$ and conductivity $\sigma$. In Section 3 we generalize the result for an arbitrary cross section of the pipe, and for a transverse impedance.

In a recent paper [3], the problems of the impedance of an insert with a different conductivity has been studied for a more general case, without the assumption (2), although for a cylindrical geometry only. In the last Section of this paper we discuss the relation of our results to that of Ref. [3].

\section{Longitudinal impedance of an insert in a round pipe}

Consider a perfectly conducting pipe which has an infinite conductivity everywhere except for a short piece of length $L$ with the wall conductivity $\sigma$ (see Fig. 1). Assume also that the pipe has a round cross section of radius $b$. Our goal is to calculate the longitudinal impedance of such pipe at frequency $\omega$. We assume that the skin depth in the metal is small compared to the pipe radius and the wall thickness, and use the Leontovich boundary condition relating the longitudinal electric field on the metal surface $E_{z}$ with the magnetic field $H_{\phi}[4]$

$$
E_{z}=-\zeta H_{\phi},
$$

where $\zeta=(1-i) \sqrt{\omega / 8 \pi \sigma}$ (we use CGS units throughout the paper). As is known, for impedance calculation, the beam can be represented as a filament 


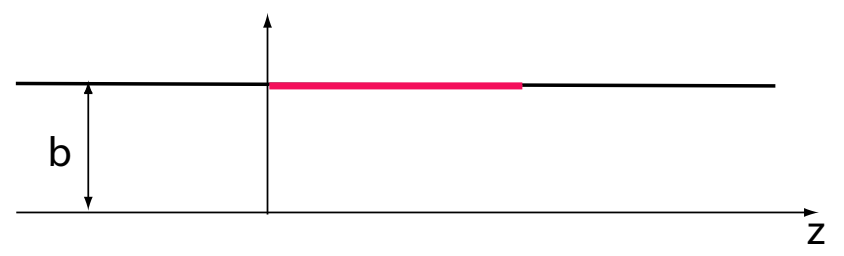

Figure 1: Round pipe with an insert of length $L$ (shown in red) having the wall conductivity $\sigma$. The rest of the pipe (shown in black) has an infinite conductivity.

current $I(z, t)$ on the axis of the pipe,

$$
I(z, t)=I_{0} e^{-i \omega t+i k z}
$$

with $k=\omega / c$. The impedance is then given by the Fourier transform of the longitudinal electric field generated by the wall currents on the axis of the pipe:

$$
Z(\omega)=-\left.\frac{1}{I_{0}} \int_{-\infty}^{\infty} E_{z}\right|_{r=0} e^{-i k z} d z,
$$

[in Eq. (5) and below, we drop the time dependent factor $\left.e^{-i \omega t}\right]$.

Our approach is based on a perturbation theory which assumes that the magnetic field on the wall of the pipe with finite conductivity is approximately equal to its value for a perfectly conducting wall:

$$
H_{\phi}=\frac{2 I_{0}}{b c} e^{i k z}
$$

Note that this is exactly the approximation that results in Eq. (1) for an infinitely long pipe [5]. When we use the relation (3) at $r=b, 0<z<L$, the mathematical problem reduces to the following one: find a solution of Maxwell's equations that satisfies the boundary condition

$$
\begin{aligned}
& \left.E_{z}\right|_{r=b}=-\zeta \frac{2 I_{0}}{b c} e^{i k z}, \quad 0<z<L, \\
& \left.E_{z}\right|_{r=b}=0, \quad z<0, \quad L<z .
\end{aligned}
$$

For calculation of the longitudinal impedance, we will only need the longitudinal component of the electric field $E_{z}(r, z)$ inside the pipe.

The solution can be found using a Green function method. We define the Green function, $G(r, z)$, as the z-component of the electric field, $E_{z}(r, z)$, satisfying Maxwell's equations with the boundary condition

$$
\left.E_{z}\right|_{r=b}=\delta(z)
$$


With this Green function the solution satisfying the boundary conditions (7) is given by

$$
E_{z}(r, z)=-\frac{2 I_{0} \zeta}{b c} \int_{0}^{L} d z^{\prime} G\left(r, z-z^{\prime}\right) e^{i k z^{\prime}} .
$$

In a round pipe, the Green function can be represented as a superposition of axisymmetric $\mathrm{TM}_{0, m}$ modes propagating in forward and backward directions in the regions of positive and negative values of $z$, respectively:

$$
G= \begin{cases}\sum_{m=1}^{\infty} a_{m}^{+} E_{z, m}^{+}, & \text {for } z>0, \\ \sum_{m=1}^{\infty} a_{m}^{-} E_{z, m}^{-}, & \text {for } z<0,\end{cases}
$$

where $E_{z, m}^{ \pm}$is the $z$-component of the electric field in the $\mathrm{TM}_{0, m}$ mode of frequency $\omega$,

$$
E_{z, m}^{ \pm}=\frac{\mu_{m}^{2}}{b^{2}} J_{0}\left(\mu_{m} \frac{r}{b}\right) e^{ \pm i k_{m} z}
$$

with $k_{m}=\sqrt{k^{2}-\left(\mu_{m} / b\right)^{2}}, J_{0}$-the Bessel function of zeroth order, and $\mu_{m}$ - the $m$ th root of $J_{0}$. We denote modes propagating in the positive and negative directions with the plus and minus signs, respectively, and define $k_{m}$ so that above the cutoff $k_{m}>0$, and below the cutoff $k_{m}$ is purely imaginary with $\operatorname{Im} k_{m}>0$.

The amplitude of each mode, $a_{m}^{ \pm}$, can be found using standard methods of electrodynamics (see, e.g., [6]):

$$
a_{m}^{ \pm}=a_{m} \equiv \frac{i}{k_{m} \mu_{m} J_{1}\left(\mu_{m}\right)} .
$$

Using Eqs. (9) and (5) we can now calculate the impedance of the insert

$$
\begin{aligned}
Z & =\frac{2 \zeta}{b c} \int_{-\infty}^{\infty} e^{-i k z} d z \int_{0}^{L} d z^{\prime} G\left(0, z-z^{\prime}\right) e^{i k z^{\prime}} \\
& =\frac{2 L \zeta}{b c} \sum_{m} \frac{a_{m} \mu_{m}^{2}}{b^{2}}\left(\int_{-\infty}^{0} e^{-i\left(k+k_{m}\right) \zeta} d \zeta+\int_{0}^{\infty} e^{-i\left(k-k_{m}\right) \zeta} d \zeta\right) \\
& =-\frac{2 L \zeta}{b c} \sum_{m} \frac{2 i k_{m} a_{m} \mu_{m}^{2}}{\left(k^{2}-k_{m}^{2}\right) b^{2}} \\
& =\frac{4 L \zeta}{b c} \sum_{m} \frac{1}{\mu_{m} J_{1}\left(\mu_{m}\right)} .
\end{aligned}
$$

Using the identity

$$
\sum_{m=1}^{\infty} \frac{1}{\mu_{m} J_{1}\left(\mu_{m}\right)}=\frac{1}{2},
$$

we arrive at the following expression for the impedance

$$
Z=\frac{2 L \zeta}{b c}=L \frac{1-i}{c b} \sqrt{\frac{\omega}{2 \pi \sigma}}
$$


which is exactly equal to $L Z_{\text {long pipe }}(\omega)$. We see that the impedance per unit length (of the resistive part) is given by the same Eq. (1) as in the case of an infinitely long pipe.

We can now justify the requirement Eq. (2). In our derivation we assumed that the magnetic field on the wall is the same as in the case of perfect conductivity. However, the magnetic field is generated not only by the beam current, but also by the displacement current,

$$
j_{z}^{\text {disp }}=\frac{1}{4 \pi} \frac{\partial E_{z}}{\partial t}
$$

(this current vanishes in the limit of perfect conductivity when $E_{z}=0$ ). To be able to neglect the corrections to $H_{\phi}$ due to $j_{z}^{\text {disp }}$, we require the total displacement current to be much less then the beam current. In the Fourier representation, the time derivative $\partial / \partial t$ reduces to multiplication by $-i \omega$, and the requirement becomes

$$
\pi b^{2} \frac{1}{4 \pi} \omega E_{z} \ll I_{0} .
$$

Using the relation $E_{z} \sim \zeta H_{\phi} \sim 2 \zeta I_{0} / b c$ we easily obtain from Eq. (17) the condition (2). Note that for very short inserts the above expression for $E_{z}$ overestimates its value and the actual requirement is looser than stated by Eq. (2). We will return to the discussion of this condition in the last section of the paper.

\section{Generalization for arbitrary cross section and transverse impedance}

The result of the previous section can be derived in a simpler way, which also allows generalization to the case of arbitrary cross section of the pipe. We now assume that the shape of the two-dimensional cross section of the pipe does not vary with $z$ and is given by equations $x=x(s)$ and $y=y(s)$ where $s$ is a parameter. We also assume that the conductivity of the pipe wall is equal to $\sigma$ for $0<z<L$ and $\sigma=\infty$ for $z<0$ and $z>L$.

First, we need to find the magnetic field $\boldsymbol{H}$ on the wall generated by the current $(4)$ in the limit of perfect conductivity $(\sigma \rightarrow \infty)$. This magnetic field is tangential to the wall and has only $x$ and $y$ components. It can be written as $\boldsymbol{H}=\boldsymbol{H}_{0}(\boldsymbol{\rho}) e^{-i \omega t+i k z}$ where $\boldsymbol{\rho}=(x(s), y(s))$ is a vector perpendicular to the $z$ axis indicating a position on the wall. With the given magnetic field on the wall, the tangential electric field is found through the Leontovich boundary condition,

$$
\begin{array}{ll}
E_{z}=\zeta \hat{\boldsymbol{z}} \cdot(\boldsymbol{H} \times \boldsymbol{n}), & \text { for } 0<z<L, \\
E_{z}=0, & \text { for } z<0, L<z,
\end{array}
$$


where $\boldsymbol{n}$ is the vector normal to the wall surface and directed into the metal, and $\hat{\boldsymbol{z}}$ is the unit vector along the $z$ axis. To find a solution of Maxwell's equations satisfying the boundary conditions (18) we, again, introduce the Green function $G(x, y, z)$ as the $z$-component of the electric field, $E_{z}(x, y, z)$, satisfying Maxwell's equations with the boundary condition

$$
\left.E_{z}\right|_{x=x(s), y=y(s)}=\delta(z) .
$$

With this Green function the solution satisfying the boundary conditions (18) is given by

$$
E_{z}(x, y, z)=-\frac{2 I_{0} \zeta}{b c} \int_{0}^{L} d z^{\prime} G\left(x, y, z-z^{\prime}\right) e^{i k z^{\prime}},
$$

and the impedance of the insert is

$$
\begin{aligned}
Z & =\frac{2 \zeta}{b c} \int_{-\infty}^{\infty} e^{-i k z} d z \int_{0}^{L} d z^{\prime} G\left(0,0, z-z^{\prime}\right) e^{i k z^{\prime}} \\
& =\frac{2 \zeta}{b c} L S
\end{aligned}
$$

with

$$
S=\int_{-\infty}^{\infty} e^{-i k \tau} d \tau G(0,0, \tau) .
$$

We see that the impedance per unit length does not depend on $L$, and hence is equal to it value in the limit of an infinitely long pipe, $L \rightarrow \infty$.

In Eq. (21) we used the Green function $G\left(0,0, z-z^{\prime}\right)$ corresponding to the zero offset of the trailing particle. Our conclusion about the ratio $Z / L$ being independent on $L$ is also valid for a more general case when the impedance is calculated with the function $G\left(x, y, z-z^{\prime}\right)$, corresponding to the trailing particle moving with the offset $(x, y)$ in the transverse direction. Through the Panofsky-Wenzel theorem [7] this longitudinal impedance is directly related to the transverse one. Hence, we conclude that the transverse impedance per unit length of an insert, as well as the longitudinal one, will also be given by the formula derived in the limit of an infinitely long pipe.

\section{Discussion}

Using the Green function technique it is straightforward to generalize our result for the case when the conductivity of the pipe is an arbitrary function of longitudinal coordinate, $\sigma=\sigma(z)$. For the round pipe, one obtains

$$
Z(\omega)=\frac{1-i}{c b} \sqrt{\frac{\omega}{2 \pi}} \int d z \sigma(z)^{-1 / 2},
$$


and for a pipe of arbitrary cross section one finds

$$
Z(\omega)=\frac{1-i}{c b} S \sqrt{\frac{\omega}{8 \pi}} \int d z \sigma(z)^{-1 / 2},
$$

with $S$ given by Eq. (22). We emphasize here that the formfactor $S$ can be obtained from the impedance calculated in the limit of an infinitely long pipe. Such impedance for a rectangular and elliptic cross sections of the pipe can be found in Ref. [8].

For a particular case of a round pipe, the impedance of a resistive insert was recently calculated in Ref. [3] for a general case of arbitrary frequencies without requiring Eq. (2). In the limit when Eq. (2) is satisfied, the result of this paper reduces to our result. Moreover, analysis of the solution in Ref. [3] shows that for very short inserts the condition of validity of Eq. (15) is less stringent than that given by Eq. (2). Specifically, the condition Eq. (2) is only necessary for $L \gg\left(\sigma b^{4} / c\right)^{1 / 3}$; in the opposite limit $L \ll\left(\sigma b^{4} / c\right)^{1 / 3}$ it should be replaced by the following one

$$
\omega \ll \omega_{0}\left(\frac{\sigma b^{4}}{c L^{3}}\right)^{1 / 6} \sim \sqrt{\frac{\sigma c}{L}} .
$$

\section{Acknowledgments}

This work was supported by Department of Energy contract DE-AC02$76 \mathrm{SF} 00515$.

\section{References}

[1] A. W. Chao, Physics of Collective Beam Instabilities in High Energy Accelerators (Wiley, New York, 1993).

[2] K. L. F. Bane and M. Sands, in E. B. Blum, M. Dienes, and J. B. Murphy, eds., Micro Bunches Workshop (AIP, New York, 1996), AIP Conference Proceedings No. 367, p. 131.

[3] S. Krinsky, B. Podobedov, and R. L. Gluckstern, Phys. Rev. ST Accel. Beams 7, 114401 (2004).

[4] L. D. Landau and E. Lifshitz, Electrodynamics of Continuous Media, vol. 8 of Course of Theoretical Physics (Pergamon, London, 1960), 2nd ed., (Translated from the Russian).

[5] G. V. Stupakov, in S. I. Kurokawa, S. Y. Lee, J. Miles, and E. A. Perevedentsev, eds., High Quality Beams. Joint US-CERN-JAPAN-RUSSIA Accelerator School (AIP, New York, 2001), AIP Conference Proceedings No. 592, pp. $205-230$. 
[6] G. V. Stupakov, Phys. Rev. ST Accel. Beams 1(6), 064401 (1998).

[7] W. K. H. Panofsky and W. Wenzel, Rev. Sci. Instr. 27, 967 (1956).

[8] R. L. Gluckstern, J. van Zeijts, and B. Zotter, Phys. Rev. E47, 656 (1993). 